\title{
LAS REPOBLACIONES DE EUCALIPTOS Y SU IMPACTO EN LA ULTIMA DECADA 1973-83
}

Dominga MARQUEZ FERNANDEZ*

\section{INTRODUCCION}

Las plantaciones de eucalipto en Andalucía y sus incidencias en el medio natural y humano, se han convertido en el último decenio en un tema polémico, controvertido y al que se le han dedicado muchas horas de trabajo. Ello se debe a las superficies que este cultivo forestal ocupa, a los teóricos beneficios obtenidos por el crecimiento rápido de la especie y a los impactos positivos y negativos que tiene sobre el medio.

En efecto, el eucalipto en Andalucía ha ocupado y está ocupando espacios que tradicionalmente han sido dedicados a unos aprovechamientos silvopastorales, creando las consiguientes modificaciones en el paisaje andaluz. El interés del tema aumenta si tenemos en cuenta que Andalucía tiene mas del $50 \%$ de la superficie total dedicada a eucaliptales en España, y estos espacios se concentran en Andalucía Occidental, mas concretamente en la provincia de Huelva.

La importancia que ha adquirido el eucalipto en nuestro país, se debe fundamentalmente a su interés económico ya que si los bosques naturales son todavía hoy la forma, casi exclusiva, de producción de madera y el consumo de la misma es creciente, no existe otra posibilidad para aumentar las disponibilidades de madera que la explotación de especies de crecimiento rápido. Si tenemos en cuenta la evolución sufrida por los espacios y la economía agrícola que libera considerables superficies de tierras de los cultivos tradicionales, se impone un nuevo ordenamiento productivo de la madera, introduciendo especies que se adecuen al medio físico y humano de estas zonas.

Ahora bien, para esta introducción masiva de nuevas especies son imprescindibles estudios previos, para que antes de modificar un ecosistema natural, se puedan preveer cuales serán las consecuencias de este nuevo cultivo, tanto en el medio físico como en el humano y poder evitar así esas disfunciones ecológicas y humanas que son irreversibles.

* Profesora Titular de Geografía Humana. Departamento de Geografía. Universidad de Sevilla. 
En el caso que nos ocupa, Andalucía y sus repoblaciones de eucaliptos, estos estudios previos no existen o son enormemente parciales y ha sido a] ver la gran modificación paisajística que Andalucía ha sufrido con los desmontes, plantaciones y cortas masivas de eucaliptos, cuando especialistas no forestales, geógrafos, ecólogos, biólogos, etc., junto con los propios campesinos, han dado la voz de alarma para que los desmontes se paralicen y, sobre todo, para que el eucalipto no se presente a la opinión pública como única alternativa económica para solucionar la miseria y marginación de extensas zonas, sino que el eucalipto se plantee como una alternativa más, al mismo nivel que la alternativa ganadera, cuya tradición es de todos conocida, sobre todo en la Sierra Morena Occidental y, en todo caso, sabiendo que las dos alternativas necesitan de fuertes inversiones, pero que mientras la primera modifica fundamentalmente el ecosistema natural, la segunda se adecua mejor a él.

Por último hay que tener en cuenta que si el móvil de las repoblaciones de eucalipto en Andalucía es su aprovechamiento económico, este criterio se mueve entre dos polaridades: los planteamientos a corto plazo, con el horizonte de la rentabilidad inmediata como dominante; y los planteamientos a largo plazo, con visión mas amplia, contemplando otros parámetros como rentabilidades indirectas. Los primeros tienden a generar desequilibrios más fácilmente que los segundos. Pues bien, en el caso de las repoblaciones con eucaliptos en Andalucía, nos encontramos dentro de un planteamiento del primer tipo lo que predispone la alteración de los ecosistemas naturales, como ya se ha probado en otras publicaciones (MARQUEZ, 1977) (AMORES, 1978) (GARCIA NOVO, 1978).

\section{SUPERFICIES DE EUCALIPTOS: EVOLUCION Y CAMBIOS EXPERIMENTADOS}

Las superficies ocupadas por las frondosas en Andalucía es en la actualidad de 1.260.914 has. de las cuales el $90 \%$ se localizan en Andalucía Occidental $^{1}$. Dentro de las especies frondosas destacamos el eucalipto, especie que en el conjunto de Andalucía Occidental supone el $40 \%$ del total de las mismas, dándose su mayor intensidad de ocupación en la provincia de Huelva. La incidencia pues, de las repoblaciones de eucalipto es masiva en Andalucía Occidental, mientras que Andalucía Oriental se ve, de momento, libre de este cultivo.

1. Las fuentes de información para la elaboración de este apartado son: Anuario de Estadísticas Agraria de 1974 y 1982 . ICONA, y datos facilitados por la Dirección Provincial del Ministerio de Agricultura de Sevilla y Huelva. 
De las especies del género Eucalyptus, en el S.O. español se cultivan fundamentalmente: Eucalyptus camaldulensis Dehn., E. viminalis labill. y E. globulus labill., por ser estas las que mejor se han adecuado al medio físico andaluz. La utilidad de estas especies se fundamenta en su crecimiento rápido, la dureza de su madera, elasticidad, fácil exfoliación, abundancia de esencias en sus hojas y existencia de tanino en la corteza, etc. Así mismo, han colaborado en la expansión del eucalipto factores tan decisivos como: reducción progresiva del ciclo de producción, aumento del volumen maderable, presencia de un mercado próximo que absorbe la producción, constante aumento del consumo de madera a nivel nacional, etc.

El análisis de las superficies ocupadas por eucaliptos, lo centraremos en la provincia de Huelva por ser dicha provincia la que soporta mayor número de has., sufre las consecuencias positivas y negativas de dichas masas forestales y han sido estudiadas con anterioridad ${ }^{2}$ lo que facilita el análisis de su evolución.

Las repoblaciones de eucalipto comienzan hacia 1941, tienen su auge en la década de los 60-70 siendo en la actualidad de 228.400 has., constatándose un retroceso a partir de 1975 , si bien algunas estadísticas oficiales no reflejan esta realidad. Como hecho expresivo de esta realidad es que durante 1984 sólo se han repoblado 600 has. de eucaliptos, siendo por el contrario importantes las superficies destoconadas.

La evolución de las superficies forestales en la provincia de Huelva en los últimos 10 años ha sido:

$$
\begin{aligned}
& 1972 \ldots 431.201 \text { has. } \\
& 1983 \ldots 577.200 \text { has. }
\end{aligned}
$$

Detectamos, pues, un aumento en dichas superficies, con una media anual de repoblaciones de 11.000 has. anuales.

En cuanto a las especies dominantes coníferas y frondosas, su evolución

\begin{tabular}{|c|c|c|c|c|}
\hline & 1983 & $\%$ & 1983 & \%o \\
\hline Frondosas . . . . . . & 329.089 has. & 76,3 & 464.400 has. & 80,5 \\
\hline Conifferas. . . . . . . & 102.112 has. & 23,7 & 112.700 has. & 19,5 \\
\hline TOTAL. . . . . . . . & 431.201 has. & 100 & 577.100 has. & 100 \\
\hline
\end{tabular}
es como sigue:

2. MARQUEZ FERNANDEZ, D.: La Geoeconomía forestal de Huelva y el dilema de los eucaliptales. I.D.R. n. ${ }^{\circ} 11$. Sevilla, 1977. pág., 63. 
Centramos nuestro análisis en las frondosas y más en concreto en la superficies ocupadas por el eucalipto, dichas masas forestales están presente a lo largo de toda la provincia, siendo escasos los municipios donde no apare cen, al menos, como elemento decorativo.

El eucalipto ha ocupado y ocupa las siguientes superficies:

$$
\begin{aligned}
& 1973 \ldots \\
& 1983 \ldots \\
& 928.070 \text { has. }
\end{aligned}
$$

En las 228.400 has. que existen hoy en la provincia de Huelva, dedicadas a eucalipto el $40 \%$ de ellas, 92.400 has. son controladas/administradas por ICONA, son las superficies pertenecientes a M. del Estado, M. de Utilidad Pú. blica y M. Consorciados, en tanto que el $60 \%$ restante y que figuran comc propiedad de particulares, pertenecen a las seis empresas forestales que actúan en la región: ENCESA, TAFISA, TORRAS HOSTENC:I, IBERFLORESTA, S.A., REFORES y ALVAREZ FORESTA. Las repoblaciones de particulares, no de empresas, han disminuido en la última década debido fundamentalmente al estancamiento de los precios de la madera y al ataque de plagas

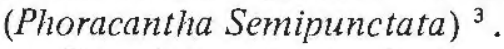

Este último hecho, la plaga Phoracantha ${ }^{3}$ se ha visto favorecida por la sequía sufrida en Andalucía en los últimos años, lo que ha forzado a la Administración a organizar campañas de lucha control, sin que los resultados hayan sido demasiado positivos. Se ha detectado más intensidad de ataque de la plaga a las plantaciones del Sur que a las del Norte de la provincia, por lo que algunos propietarios han realizado destoconado de los eucaliptos, introduciendo otros cultivos, tales como: naranjos, almendros, y últimamente fresas. Cultivos que cuentan con una mayor demanda y cierta seguridad de precios en el mercado, tanto nacional como internacional. A lo largo de 1984 se han sustituido por naranjos 1.000 has. y en torno a 2.000 de fresas.

La situación actual en cuanto a localización de las masas de eucaliptos, su producción y su problemática, es como sigue:

- Descenso progresivo de las repoblaciones.

- Localización de las grandes masas en la zona Norte de la provincia.

- Introducción de nuevos cultivos en zonas ocupadas antes por eucaliptos.

- Control progresivo de la plaga Fhoracantha y prohibición de comercializar la madera de eucalipto fuera de Andalucía, para evitar mayores contaminaciones.

3. CADAHIA, D.: "Proximidad de los nuevos enemigos de los eucaliptos en España". Bol. Investigación y Técnica del papel. 1981. MARTINEZ EGEA, J.M.: "Phoracantha Semipunctata. FAB. en el S.O. español. Resumen de la campaña de colocación de árboles de cebo". Bol. de la Estación Central de Ecología. Vol. 11, n. ${ }^{\circ}$ 2. Madrid, 1982. 
- Descenso de la mano de obra ocupada en el sector, por la progresiva mecanización del cultivo del eucalipto.

- Por tanto descenso de la rentabilidad de los eucaliptales existentes motivada por la dificultad de comercializar la madera, por la subida de precios de la maquinaria, lo que provoca un estancamiento o disminución de las superficies dedicadas a eucaliptos.

\section{IMPACTOS DEL EUCALIPTO}

Analizadas las superficies ocupadas por las masas forestales de eucaliptos, esbozaremos ahora los impactos que este cultivo con especies alóctonas han supuesto y están suponiendo, tanto en el medio físico como en el humano.

En el medio físico la incidencia es múltiple, pero hemos de destacar como más importantes las siguientes:

- Alteración del suelo. Efectivamente la capa superficial de la tierra que soporta este tipo de cultivo se ve trasformada tanto en profundidad, sobre todo en la composición del pH que disminuye y como consecuencia se acidifica, como superficialmente ya que las cortezas que se desprenden de los árboles, por su composición, no favorecen el proceso de humificación y sí el de mineralización, con el consiguiente empobrecimiento bioIógico de la capa superficial, desapareciendo, después de los primeros años, todo tipo de sotobosque. El eucalipto empobrece el suelo ya que toma de él los nutrientes sin la contrapartida de la humificación por no existir organismos detrivoros capaces de transformar la hojarasca del eucalipto en materia órganica y mineral aprovechables para el suelo.

- Alteración en el régimen hidrológico. Varios son los factores que inciden en el régimen hidrológico de zonas preparadas para eucaliptales, mas Ia consecuencia es única: disminuye la cantidad de agua disponible para otros usos. En ello influye: el sistema radicular de la especie que penetra a gran profundidad hasta llegar a las capas freáticas, la supresión del suelo y la vegetación lo que da lugar a la inestabilidad en el sustrato. Esta es una acción sobre las aguas subterráneas, pero el eucalipto también modifica el volumen de las aguas superficiales, ya que se ha observado que en zonas próximas a plantaciones de eucaliptos se agotan los manantiales, se secan los arroyos, etc., de aquí que los eucaliptales se utilicen frecuentemente para la descación de zonas pantanosas. 
- Efectos del aterrazado. El efecto mayor sobre la morfología de las zona: cultivadas de eucaliptal viene dada por la construcción de terrazas par: la plantación, en las zonas de pendiente. Con esta acción y la consiguien te desaparición de la vegetación natural, aumenta considerablemente le erosión. La construcción de terrazas sobre zonas de pendiente lleva consi go el que los taludes debido a su pendiente y a su naturaleza poco com. pacta, sufran una erosión mucho mayor que la sufrida por la ladera origi. naria. Respecto al trazado de terrazas horizontales la dificultad mayor es. triba en que no se desarrolla una red de drenaje y en caso de precipitacio. nes intensas el agua circula transversalmente por las terrazas, saltando d $\epsilon$ talud en talud. Este fenómeno tiende a ampliarse transportando materia. les finos, siendo este mecanismo el que explica la contaminación de cau. ces de agua próximos a zonas repobladas.

En resumen, las influencias del eucaliptal en el medio físico, son siempre negativas, teóricamente, jamás indiferentes y rara vez positivas.

En el medio biológico los impactos del eucalipto son también importantes y los tratamos de evaluar a través de las modificaciones sufridas tanto por la flora como por la fauna de los espacios ocupados por dicha especie forestal. Como indicadores de las transformaciones sufridas utilizaremos las plantas autóctonas vegetales y, repecto a la fauna, las aves que anidan o viven en dichas formaciones y los mamíferos, por entender que dichas especies son las que más variación sufren dentro del ecosistema donde se instalan los eucaliptos.

De forma esquemática podemos afirmar que en el medio biológico el eucalipto provoca ${ }^{4}$ :

- Empobrecimiento de plantas autóctonas, ya que elimina el matorral en un radio, mas o menos aproximado, de dos metros de su tronco. Desaparecen las especies muy heliofilas, ya que el eucalipto como árbol produce una sombra escasa. La regeneración de la vegetación natural se hace difícil por el afecto alopático de los aceites esenciales de las hojas del eucalipto.

- Empobrecimiento de la fauna. Por una parte desaparecen las especies que dependen del agua, quedando reducidas a zonas de barrancos húmedos donde aún quedas setos.

- Por otra parte hay empobrecimiento de consumidores primarios (fitófagos) y los consumidores secundarios, al desaparecer los primeros, también desaparecen.

4. AMORES, F.: Repercusiones de las repoblaciones de eucalipto en la fauna. Actas de las Jomadas del Eucalipto celebradas en Huelva. 1978, pág. 125. GARCIA NOVO, F.: Impacto de las plantaciones de eucaliptos. Actas de las jornadas del eucalipto. Huelva, 1978, pág. 33. 
Desde el punto de vista humano las repoblaciones de eucalipto también provocan modificaciones. Se ha querido hacer ver que el eucalipto, por la mano de obra que necesita, puede ser considerado como un cultivo social, pero esto no es así, ya que si bien este cultivo en un primer momento necesita mano de obra abundante para las labores de preparación del terreno, plantación, etc., sin embargo es escasa la mano de obra necesaria en las labores intermedias por ser pocas las que se realizan y ser su corta mecanizada, así como su carga y transporte.

Este hecho nos permite deducir que el cultivo del eucalipto no supone una mejora en las condiciones de vida del campesino, ni por tanto constituye un medio efectivo para luchar contra el paro de nuestra región. Estudios realizados ponen de manifiesto que para un puesto de trabajo fijo en el sector forestal, hacen falta unas 95 has. de eucaliptal en tanto que en las mismas zonas, en una actividad ganadera son necesarias unas 70 has., para la creación de un puesto de trabajo. De aquí que para algunos autores este cultivo es no sólo antisocial, sino que, a la vez, provoca la emigración de la mano de obra joven con lo cual los pueblos disminuyen su población y se aprecia un envejecimiento de la misma.

Cabría mencionar también la falta de aprovechamientos secundarios de estos espacios forestales, tales como una ganadería o una actividad cinegética subsidiaria que podría proporcionar a la economía de los medianos agricultores unos ingresos que les ayudaran a paliar la crisis que sufre la agricultura tradicional. Esto no es posible por el empobrecimiento de la flora que hemos expuesto anteriormente.

Si toda la acción del hombre sobre el medio produce la modificación de los ecosistemas naturales, en el caso de las repoblaciones de eucalipto las modificaciones abarcan todos los medios posibles, ya que aunque en teoría las repoblaciones son menos agresivas con el medio que la agricultura, el eucalipto hay que considerarlo como un verdadero cultivo, que transforma paisajes, modifica los modos de vida de las comarcas repobladas y por tanto, modifica los ecosistemas.

En los espacios forestales del Mediterráneo los aprovechamientos han sido destructivos: leña, carboneo, sobrepastoreo, desmonte, etc., y hoy las prácticas repobladoras con especies foráneas, amenazan con la sustitución de los reductos forestales de arbolado natural. El móvil es exclusivamente económico y no median otras consideraciones o análisis.

Sin embargo cuando las repoblaciones van a espacios ya degradados o poco rentables la práctica adquiere perspectivas diferentes, mas favorables.

La vida de la humanidad, si pensamos ecológicamente, ha de apoyarse en el aprovechamiento racional y científico de los ecosistemas naturales, cuya reducción o transformación entrañan serios peligros. No sólo no sabemos aprovechar su productividad, sino que se está empezando a desvelar cuál es. 
Estabilidad y productividad se maximizan en la naturalezs no sólo por cantidad bruta, sino por cantidad unido a variedad. Cantidad sóla puede maximizarse a costa de desequilibrio, por lo tanto, riesgo. Ante ello cabe preguntar ¿puede volverse a una situación de equilibrio? Sabemos que en situaciones límites, como en el caso del bosque Mediterráneo, no.

La acción del hombre sobre el paisaje vegetal natural es y ha sido grande a través del tiempo; abarca desde la protección y potenciación del paisaje existente por medio de la tecnificación, hasta la transformación total del mismo, mediante las repoblaciones forestales con distintas especies no autóctonas, cuya fisonomía nada tiene que ver con el paisaje primitivo precedente, como es el caso de las plantaciones con eucaliptos.

El hombre en sus roturaciones, agricultura, ha cambiado un ecosistema por otro que se mantiene y persiste por la acción y para la utilidad del hombre. El paso del tiempo a lo largo de la historia se ha encargado de demostrar la viabilidad de muchas de estas transformaciones ecológicas realizadas empíricamente y muestra de su éxito puede ser la posibilidad de alimentar a una humanidad creciente como la actual. Pero también tenemos la evidencia de otras muchas transformaciones no acertadas y con el problema de la irreversibilidad de los procesos destructivos realizados por el hombre en espacios naturales cuyo potencial biológico se mantiene en equilibrio precario. 